\title{
Phase prediction method for pattern formation in time-dependent Ginzburg-Landau dynamics for kinetic Ising model without a priori assumptions of domain patterns
}

\author{
Ryoji Anzaki $\odot,{ }^{1}$ Shin-ichi Ito $\odot,{ }^{1,2}$ Hiromichi Nagao $\odot,{ }^{1,2, *}$ Masaichiro Mizumaki, ${ }^{3}$ Masato Okada, ${ }^{4}$ and Ichiro Akai ${ }^{5}$ \\ ${ }^{1}$ Earthquake Research Institute, The University of Tokyo, 1-1-1, Yayoi, Bunkyo-ku, Tokyo 113-0032, Japan \\ ${ }^{2}$ Graduate School of Information Science and Technology, The University of Tokyo, 7-3-1, Hongo, Bunkyo-ku, 113-8656 Tokyo, Japan \\ ${ }^{3}$ Japan Synchrotron Radiation Research Institute (JASRI/SPring-8), 1-1-1 Kouto, Sayo, Hyogo 679-5198, Japan \\ ${ }^{4}$ Graduate School of Frontier Sciences, The University of Tokyo, Kashiwa, Chiba 277-8561, Japan \\ ${ }^{5}$ Institute of Industrial Nanomaterials, Kumamoto University, 39-1 Kurokami 2, Kumamoto 860-8585, Japan
}

(Received 15 September 2020; revised 10 February 2021; accepted 15 February 2021; published 4 March 2021)

\begin{abstract}
We propose a phase prediction method for pattern formation in a two-dimensional kinetic Ising model with dipole-dipole interactions under the time-dependent Ginzburg-Landau dynamics. Considering the effects of the material thickness by assuming uniformness along the magnetization axis, the model corresponds to thin magnetic materials with long-range repulsive interactions. We formulate a theoretical basis to understand the effects of the material parameters on the formation of the magnetic domain patterns in terms of the equilibrium equations governing the balance between the linear and nonlinear forces in the equilibrium state. Further, we develop a method for predicting the phase in the equilibrium state achieved after the dynamical evolution of a system with given initial parameters. The analytically hard third-order term is approximated using the restricted phase-space approximation [Anzaki et al., Ann. Phys. (NY) 353, 107 (2015)] for the $\phi^{4}$ models. Although the proposed method does not have perfect concordance with the actual numerical results, it has no arbitrary parameters and functions to tune the prediction. In other words, it is a method with no a priori assumptions of the domain patterns.
\end{abstract}

DOI: 10.1103/PhysRevB.103.094408

\section{INTRODUCTION}

Magnetic materials were of great interest even before the beginning of the application of quantum physics in solid-state physics [1]. Domain patterns are essential for understanding magnetic materials because the macroscopic properties of magnetic materials are largely affected by domain patterns [2]. In light of the recent progress in experimental methods for observing magnetic domain patterns, information on the magnetic dynamics can be obtained from domain patterns, e.g., the material parameters, external magnetic fields, and size of the magnetic materials. X-ray magnetic circular dichroism [3] and the detection of the Kerr effect [4] via visible light [5] are well-known experimental methods for detecting magnetization. In the realm of theoretical and simulation physics, researchers have already made progress towards this aim. Jagla [6] and Kudo and Nakamura [7] performed numerical simulations using similar models to reproduce the magnetic domain patterns on two-dimensional magnetic materials. The latter proposed a relation between the sweep rate of the external magnetic field and the final magnetic domain patterns in the equilibrium state. They utilized a two-dimensional kinetic Ising spin system with spins on the square lattice lying on the $x y$ plane, while magnetization was restricted in the $z$ direction, which is normal to the $x y$ plane. Assuming that the high wave number components of the Green's function of the

*Corresponding author: nagaoh@eri.u-tokyo.ac.jp dipole-dipole interaction play a minor role, they succeeded in explaining the various domain patterns resulting from different sweep rates by solving the time-dependent GinzburgLandau (TDGL) equation numerically [7]. Iwano et al. [8] adopted a numerically evaluated effective two-dimensional Green's function for the dipole-dipole interaction.

In the early history of the research on TDGL dynamics, the probability density functions of the spin systems under TDGL dynamics were studied by Kawasaki [9-11] in the 1970s. Suzuki and Igarashi [12] also studied the same system using the Markov chain method. Their major objective was to obtain the global characteristics of the spin configuration, e.g., the dynamic magnetic susceptibility [9] and critical exponents [12], using analytical tools, such as diagrammatic methods. In the 1980s, Grant et al. [13] investigated a similar system in the context of phase separation and developed a theory using the spatial wave numbers of the fields. On the other hand, Kawasaki and Ohta [14] also investigated the kink dynamics to the one-dimensional TDGL model and those study findings were inherited to research on the dynamical phase transition in the TDGL dynamics of the XY model [14-16]. In the late 1980s, growing computational resources enabled numerical simulations to solve the TDGL equation in real space [17].

In the realm of magnetic materials, explanations of the magnetic domain patterns have been developed for decades [18-20]. The Kooy-Enz model [21] and its variants [19,22] assume simple domain patterns specified by functions with one or more parameters and minimize the total energy (the 
sum of the contributions from the domain and domain wall) with respect to the parameters. The forms of the functions that determine the domain patterns are chosen a priori to simplify the entire problem into an optimization problem of real functions. Garel and Doniach [23] analyzed the behavior of a similar system under a finite temperature $T$ and external magnetic field $H$ thermodynamically and plotted the $T-H$ phase diagram with three phases labeled uniform, bubble, and striped. These phases were also defined using simple analytic functions with few parameters.

In this study, we adopt a strategy that does not involve any a priori defined functions. The effects of the material thickness and other parameters on the TDGL pattern formation are explained using the equilibrium equation that describes the balances between the linear and nonlinear forces in the equilibrium state reached after appropriate numerical time evolutions with a realistic initial condition. This equation enables us to predict the phase that a specific TDGL equation with given system parameters forms in the equilibrium state. In other words, we can predict the magnetic domain pattern formed in thin magnetic materials for given system parameters using the proposed method.

Herein, we use a numerical method to construct an effective two-dimensional Green's function by analytically averaging the dipole-dipole interactions along the $z$ direction for each grid point on the $x y$ plane, as proposed by [8], enabling us to consider the effects of the thickness more precisely.

\section{MODEL AND METHODS}

We utilize the Ising-like spin model with TDGL dynamics $[7,13,17,24,25]$, which is also referred to as the kinetic Ising model [26]. We prepare an array of complex variables $\{\phi(r)\}$, where $\boldsymbol{r}$ is an element of two-dimensional discrete space $\mathcal{D}=\{(x, y): 1 \leqslant x, y \leqslant L\} \subset \mathbf{N}^{2}$ for a positive integer $L$. Each variable $\phi$ is regarded as a magnetic dipole restricted to the $z$ direction, while the vector $\boldsymbol{r}$ represents a coordinate on the $x y$ plane, normal to the $z$ axis. Note that the $x$ and $y$ components of the spins are set to zero in this model. By introducing the saturation magnetization, $\rho>0$, the TDGL equation for the spin system above with time parameter $t$ is expressed as follows:

$$
\frac{d \phi(\boldsymbol{r})}{d t}=W(\boldsymbol{r} \mid \phi]+B(t)
$$

where $B$ represents the explicitly time-dependent external magnetic field (restricted to the $z$ direction) and $W(\boldsymbol{r} \mid \phi]$ is a function of $\boldsymbol{r}$ and a functional of $\phi$, which is defined as follows:

$$
\begin{gathered}
W(\boldsymbol{r} \mid \phi]=\alpha\left[\phi(\boldsymbol{r})-\rho^{-2} \phi^{3}(\boldsymbol{r})\right]+\beta \nabla^{2} \phi(\boldsymbol{r})-\gamma F[\phi], \\
F[\phi]=\int d^{2} r^{\prime} G\left(\boldsymbol{r}-\boldsymbol{r}^{\prime}\right) \phi\left(\boldsymbol{r}^{\prime}\right) .
\end{gathered}
$$

The terms containing $\alpha, \beta$, and $\gamma$ correspond to the anisotropy, exchange, and dipole-dipole interactions, respectively. The last term is represented by Green's function for the magnetic dipole-dipole interaction $G$.
By moving into the wave number space using the (nonunitary) Fourier transform

$$
\langle f\rangle_{\boldsymbol{k}}=L^{-2} \sum_{\boldsymbol{r} \in \mathcal{D}} f(\boldsymbol{r}) e^{i \boldsymbol{k} \cdot \boldsymbol{r}},
$$

the TDGL above becomes

$$
\frac{d \phi_{k}}{d t}=W_{k}[\phi]+B_{k}(t)
$$

where $W_{k}[\phi], \phi_{k}$, and $B_{k}$ represent the spatial Fourier transformations of $W(\boldsymbol{r} \mid \phi], \phi(\boldsymbol{r})$, and $B(t)$, respectively. By performing the Fourier transform, we obtain the following:

$$
W_{k}[\phi]=\alpha\left\langle\phi-\frac{\phi^{3}}{\rho^{2}}\right\rangle_{k}-\beta|\boldsymbol{k}|^{2} \phi_{k}-\gamma L^{2} G_{k} \phi_{k} .
$$

Here, the Fourier transformation of $G$ is introduced via the convolution theorem, and the prefactor $L^{2}$ is associated with the choice of the Fourier transform in Eq. (4).

The effects of the thickness are not apparent but are introduced via the Fourier transform of the Green's function of the dipole-dipole interaction $G_{k}$, as already performed by [8]. Hereafter, we assume that the spin variables have the same value along the $z$ direction for each $\boldsymbol{r}$. The thickness (spatial extension along the $z$ direction) of the material is a positive value, i.e., $A>0$. By introducing the virtual $z$ coordinate $0 \leqslant z \leqslant A$, we define an effective two-dimensional Green's function under the conditions specified above as follows:

$$
G(\boldsymbol{r} ; A)=\frac{1}{A^{2}} \int_{0}^{A} d z \int_{0}^{A} d z^{\prime} \mathcal{G}\left(\boldsymbol{r} ; z-z^{\prime}\right),
$$

with the classical dipole-dipole interaction

$$
\mathcal{G}(\boldsymbol{r} ; \Delta z)=\frac{1}{\left(|\boldsymbol{r}|^{2}+\Delta z^{2}\right)^{3 / 2}}-\frac{3 \Delta z^{2}}{\left(|\boldsymbol{r}|^{2}+\Delta z^{2}\right)^{5 / 2}} .
$$

The integral (7) can be performed analytically, and

$$
G(\boldsymbol{r} ; A)=\frac{2}{A^{2}}\left(\frac{1}{|\boldsymbol{r}|}-\frac{1}{\sqrt{|\boldsymbol{r}|^{2}+A^{2}}}\right) .
$$

It should be noted that, in the limit $A \rightarrow 0, G(\boldsymbol{r})$ converges to the inverse-cubic law, pointwise.

One may consider the continuum limit, which corresponds to the case where the correlation length measured in the unit of the grid spacing becomes positive infinity. In such a case, the Fourier transform of Green's function is obtained from the real-space function $G$ and has the following analytical form:

$$
G_{k}(A)=\frac{1}{\pi A^{2}} \frac{1-e^{-A|k|}}{|\boldsymbol{k}|} .
$$

The weight of the Fourier transformation is taken to be $(2 \pi)^{-d}$, where $d=2$ is the spatial dimension. In this limit, the right-hand side of the equation of motion, Eq. (6), becomes

$$
W_{k}[\phi]=\alpha\left\langle\phi-\frac{\phi^{3}}{\rho^{2}}\right\rangle_{k}-\beta|\boldsymbol{k}|^{2} \phi_{k}-\gamma(2 \pi)^{2} G_{k}(A) \phi_{k} .
$$

Note that this representation is formally obtained simply through a replacement $L \rightarrow 2 \pi$. 


\section{NUMERICAL SIMULATIONS}

In the $x y$ plane, we use the nonunitary fast Fourier transform (FFT) corresponding to Eq. (4) to construct the modes, $\phi_{k}=\langle\phi\rangle_{k}$, and the wave number representation of Green's function $G_{k}$. We adopt the periodic boundary condition for the $x$ and $y$ directions. Hence, the entire topology of the simulation space is a torus. The spacing of the grid on the $x y$ plane is set to unity. We introduce the randomness of the coefficient of the anisotropy as $\alpha \rightarrow \alpha \Lambda(\boldsymbol{r})$, with $\Lambda(\boldsymbol{r})=1+\lambda(\boldsymbol{r}) / 4$, and the Gaussian noise as $\lambda(\boldsymbol{r}) \sim \mathcal{N}\left(0,0.3^{2}\right)$ independently and identically for all $\boldsymbol{r}$, as demonstrated by [7]. The external magnetic field intensity is represented by the rectified linear unit (ReLU) function, $R(x)=\max (x, 0)$, as $B(t)=R\left(B_{0}-v_{\mathrm{B}} t\right)$, with $B_{0}, v_{\mathrm{B}} \geqslant 0$.

The initial spin configuration is prepared in real space by distributing $\phi(\boldsymbol{r})$ randomly in the range $-1.1 \leqslant \phi(\boldsymbol{r}) \leqslant-1.0$ using a uniform distribution. The equation of motion is realized in the wave number space to achieve low computational costs for a large system using FFT. The resulting spin configuration in the next step is then moved back to real space using the inverse FFT. The computationally heavy tasks, including the convolutions of the modes $\phi_{k}$ in the cubic term, are now circumvented by this method simply by performing the algebraic operation $\phi(\boldsymbol{r}) \mapsto[\phi(\boldsymbol{r})]^{3}$ for each $\boldsymbol{r} \in \mathcal{D}$. The time evolutions are performed efficiently using the secondorder exponential time differencing fourth-order Runge-Kutta (ETD2/RK4) method [27], which is a multistep exponential integrator method, with a relatively large time step $\delta t=0.2$. The scalability to the system size $L^{2}$ is quite good, with the computational time roughly proportional to $L^{2}$, up to the largest case considered here $(L=512)$. In Fig. 1 , one can see qualitatively different final magnetic domain patterns depending on the different values of the thickness $A$. Throughout this paper, the unit of thickness is assumed to be the grid spacing in the $x y$ plane.

In this system, we can define the two-dimensional translational and rotational symmetry in the coordinate space and the $\mathbf{Z}_{2}$ symmetry of the spin. Therefore, the patterns above can be naturally classified into four phases according to the following symmetries: symmetric [Fig. 1(a)], T-breaking [Fig. 1(b)], TZ-breaking [Fig. 1(c)], and Z-breaking [Fig. 1(d)] phases, with $\mathrm{T}$ standing for "translational and rotational" and $\mathrm{Z}$ standing for $\mathbf{Z}_{2}$. Note that in the symmetric phase, $\phi(\boldsymbol{r})=0$ for all $\boldsymbol{r} \in \mathcal{D}$ because it is the only solution that is symmetric in both $\mathbf{Z}_{2}$ and translational and rotational symmetries. Previous studies (e.g., $[6,7,28])$ used more descriptive terms, such as "labyrinth" and "sea island," when referring to Figs. 1(b) and $1(\mathrm{c})$, respectively.

\section{NORMALIZATION OF THE TDGL DYNAMICS}

The physical or dimensionful TDGL equation [Eq. (1)] is normalized using the linear coordinate scale transformations to compare with other results. One of the most convenient choices is to eliminate the dimensionful saturation magnetization $\rho$. In this case, using the time variable $\tau$, spatial coordinate $\zeta$, Laplacian $\partial^{2}$, and magnetization $\varphi$, we find that the normalized TDGL equation for the dynamics under the external magnetic field swept from $B_{0}>0$ to zero at a
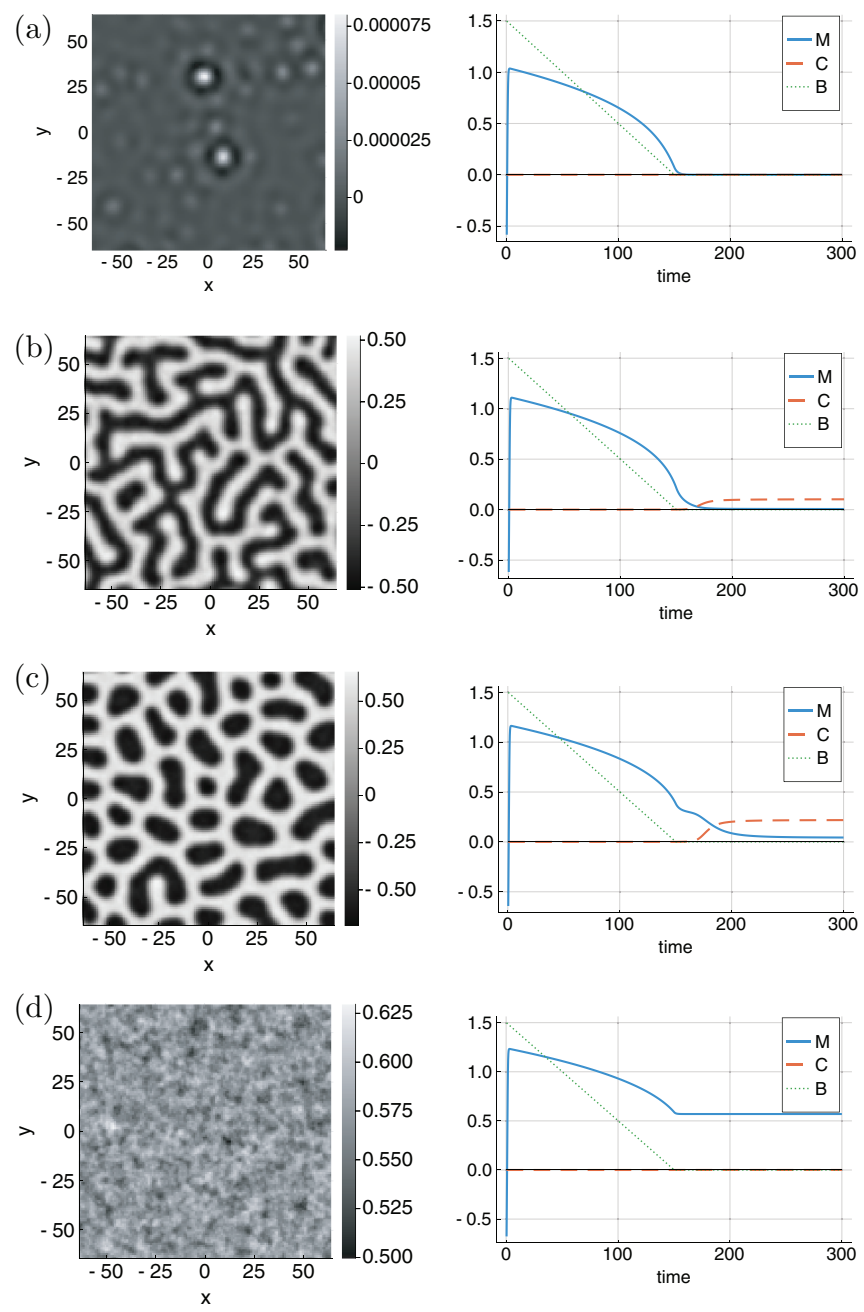

FIG. 1. Left: The magnetic domain patterns at the end of the simulation for various values of $A$. Right: The time dependences of the external magnetic field (marked $\mathrm{B}$ ), the average magnetization $\langle\phi\rangle_{0}$ (marked M), and the correlation $\left\langle\delta \phi^{2}\right\rangle_{0}$ (marked C). The simulation size is $L=512$; the duration of the time evolution is $t_{\max }=300$, and the external magnetic field intensity is $B(t)=$ $R\left(B_{0}-v_{\mathrm{B}} t\right)$, with $B_{0}=1.5, v_{\mathrm{B}}=0.01$, and $R(x)=\max (x, 0)$. The system parameters are $\alpha=1, \beta=1, \rho=1, \gamma=0.2$. The white regions have positive magnetization, whereas the black regions have negative magnetization.

constant sweep rate $v_{\mathrm{B}}$ is as follows:

$$
\begin{gathered}
\frac{d \varphi}{d \tau}=\varphi-\varphi^{3}+\partial^{2} \varphi-p_{1} f[\varphi]-R\left(p_{2}-p_{3} \tau\right), \\
f[\varphi]=\int d^{2} \zeta^{\prime} G\left(\zeta-\zeta^{\prime}\right) \varphi\left(\zeta^{\prime}\right) .
\end{gathered}
$$

Here, the linear functional $F$ denotes the dipole-dipole interaction, while the coefficients $p_{i}(i=1,2,3,4)$ are defined as follows:

$$
p_{1}=\frac{\gamma}{\sqrt{\alpha \beta}}, \quad p_{2}=\frac{B_{0}}{\sqrt{\rho \alpha}}, \quad p_{3}=\frac{v_{\mathrm{B}}}{\rho \alpha^{2}}, \quad p_{4}=\sqrt{\frac{\alpha}{\beta}} A .
$$

The last parameter $p_{4}$ represents the normalized thickness and is used to construct the effective two-dimensional Green's function, as demonstrated in Eq. (7). 


\section{EQUILIBRIUM EQUATIONS}

If the spin configuration $\phi$ is in the equilibrium state, $\dot{\phi}_{k}=0$ for all $\boldsymbol{k}$, the generic (whether it is normalized or not) equation of motion [Eqs. (1) and (2)] is simplified into a set of simultaneous time-independent equations. We now introduce the equilibrium equation, which is the equation of motion in the equilibrium state assuming zero external magnetization:

$$
\left\langle\phi^{3}\right\rangle_{k}=Q_{k}\langle\phi\rangle_{k},
$$

with

$$
Q_{k}=\frac{\alpha-\beta|\boldsymbol{k}|^{2}-\gamma L^{2} G_{k}}{\alpha} .
$$

Let us rewrite Eq. (15) in the average magnetization $\langle\phi\rangle_{0}$ and modes $\langle\delta \phi\rangle_{\boldsymbol{k}}$ with $\delta \phi(\boldsymbol{r})=\phi(\boldsymbol{r})-\langle\phi\rangle_{0}$. By noting that $\langle\delta \phi\rangle_{0}=0$ and $\langle c\rangle_{k}=0$ for any constant $c$ and $\boldsymbol{k} \neq 0$, we immediately obtain the relations governing the balance between the first-, second-, and third-order moments of the field variables at the equilibrium state. For $\boldsymbol{k}=0$,

$$
\left[Q_{0}-3\left\langle\delta \phi^{2}\right\rangle_{0}\right]\langle\phi\rangle_{0}=\left\langle\delta \phi^{3}\right\rangle_{0}+\langle\phi\rangle_{0}^{3},
$$

and for $\boldsymbol{k} \neq 0$,

$$
\left[Q_{k}-3\langle\phi\rangle_{0}^{2}\right]\langle\delta \phi\rangle_{k}=\left\langle\delta \phi^{3}\right\rangle_{k}+3\left\langle\delta \phi^{2}\right\rangle_{k}\langle\phi\rangle_{0} .
$$

These equations form a system of cubic equations that cannot be solved analytically.

\section{CATEGORIZATION OF EQUILIBRIUM EQUATIONS WITH THE RESTRICTED PHASE-SPACE APPROXIMATION}

The four types of phases mentioned in Sec. III (i.e., Z-, $\mathrm{T}-$, and TZ-breaking and symmetric phases) correspond to the types of equilibrium equations: equilibrium equations that prohibit nonzero uniform modes $\phi_{0} \neq 0$ correspond to either T-breaking or symmetric, while those that allow nonzero uniform modes correspond to either $\mathrm{Z}$ or TZ-breaking. The equilibrium equations that correspond to either T-breaking or symmetric phases are distinguished by the fact that T-breaking phases have nonzero modes, i.e., $\phi_{\boldsymbol{k}} \neq 0$ for $\boldsymbol{k} \neq 0$.

To deal with this classification problem of equilibrium equations, we now apply the restricted phase-space approximation (RPSA) [29] to the equation above. In our current context, it is equivalent to a factorization of the higher moments of $\delta \phi$ described by

$$
\left\langle\delta \phi^{3}\right\rangle_{\boldsymbol{k}} \rightarrow 3\left\langle\delta \phi^{2}\right\rangle_{0}\langle\delta \phi\rangle_{\boldsymbol{k}} \quad(\boldsymbol{k} \neq 0) .
$$

In general, the RPSA truncates the $\phi^{4}$-interaction terms systematically, and it is known to be exact in special models [29]. RPSA is an elevated version of the mean-field theory because the former contains the variance $\left\langle\delta \phi^{2}\right\rangle_{0}$ as well as the mean-field contributions.

In the diagrammatic notation, the RPSA is a restriction of the convolution in the Ginzburg-Landau pseudofree energy [corresponding to the equation of motion, Eq. (6)], as shown below. Note that summations over repeated indices are as-
TABLE I. Classification of RPSA equilibrium equations.

\begin{tabular}{lcc}
\hline \hline Type & Condition(s) & Phase(s) \\
\hline C1 & $\max _{k}\left(Q_{k}\right)<0$ & Symmetric \\
C2 & $\max _{k}\left(Q_{k}\right) \geqslant 0$ and $Q_{0}<0$ & T-breaking \\
C3 & $\max _{k \neq 0}\left(Q_{k}\right) \geqslant 0$ and $Q_{0} \geqslant 0$ & TZ or Z-breaking \\
\hline \hline
\end{tabular}

sumed in the diagrams.

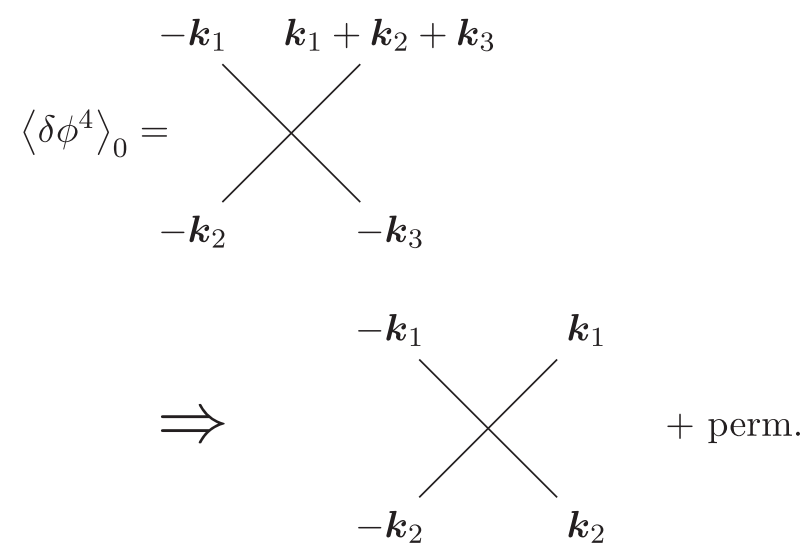

In Eq. (20), perm. indicates the permutations of the vertices. Noting that $\langle\delta \phi\rangle_{0}=0$, it must be emphasized that the RPSA approximates $\left\langle\delta \phi^{3}\right\rangle_{0}$ by zero. Thus, we obtain the RPSA equilibrium equations as shown below. For $\boldsymbol{k}=0$,

$$
\left[Q_{0}-3\left\langle\delta \phi^{2}\right\rangle_{0}\right]\langle\phi\rangle_{0}=\langle\phi\rangle_{0}^{3} .
$$

For $\boldsymbol{k} \neq 0$,

$$
\left[Q_{k}-3\langle\phi\rangle_{0}^{2}-3\left\langle\delta \phi^{2}\right\rangle_{0}\right]\langle\delta \phi\rangle_{k}=3\left\langle\delta \phi^{2}\right\rangle_{k}\langle\phi\rangle_{0} .
$$

We can then classify the RPSA equilibrium equations using the profiles of the function $Q_{k}$, as shown in Table I.

\section{RELEVANCE OF RPSA IN NUMERICAL DATA}

We check whether the numerical data of $\langle\phi\rangle_{0},\left\langle\delta \phi^{2}\right\rangle_{0}$, and $\left\langle\delta \phi^{3}\right\rangle_{0}$ satisfy the RPSA-approximated relation [Eq. (21)]. To be clear, we compare $\left\langle\phi^{\text {num }}\right\rangle_{0}$ obtained by the numerical time evolutions via the TDGL equation [Eq. (1)] with the quantity defined by the following expression:

$$
\begin{aligned}
& \left\langle\phi^{\mathrm{RPSA}}\right\rangle_{0} \\
& \quad=\left\{\begin{array}{cl}
0 & \left(Q_{0}-3\left\langle\left(\delta \phi^{\mathrm{num}}\right)^{2}\right\rangle_{0} \leqslant 0\right), \\
\sqrt{Q_{0}-3\left\langle\left(\delta \phi^{\mathrm{num}}\right)^{2}\right\rangle_{0}} & \left(Q_{0}-3\left\langle\left(\delta \phi^{\mathrm{num}}\right)^{2}\right\rangle_{0}>0\right) .
\end{array}\right.
\end{aligned}
$$

The result for the relatively small $(L=128)$ system with $\alpha=3.5, \beta=2.0$, and $\gamma=2 / \pi$ for the external field specified by $B_{0}=1.5$ and $v_{\mathrm{B}}=0.01$ is shown in Fig. 2. Figure 2 shows very good agreement between $\left\langle\phi^{\mathrm{RPSA}}\right\rangle_{0}$ and $\left\langle\phi^{\mathrm{num}}\right\rangle_{0}$. See Appendixes $\mathrm{B}$ and $\mathrm{C}$ for the validity of the numerical parameters used in this study.

This result shows that the non-RPSA effects are relatively small for these system parameters $\left(\alpha, \beta, \gamma, B_{0}, v_{\mathrm{B}}\right)$. The corresponding normalized parameters are $\left(p_{1}, p_{2}, p_{3}, p_{4}\right)=$ $(0.2406,0.8018,0.0008163,1.323 A)$ for each $A$. 

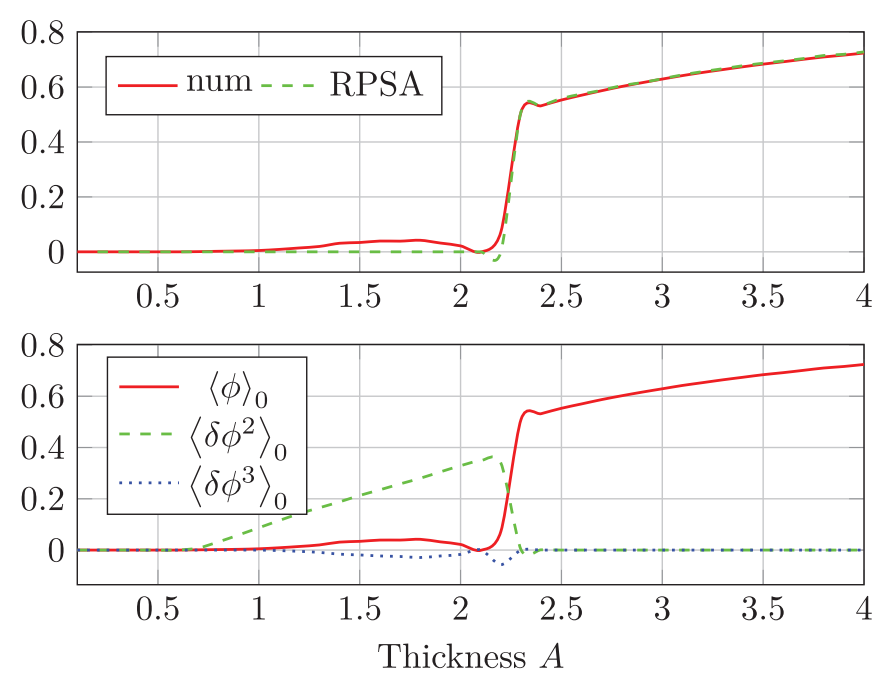

FIG. 2. A demonstration of the RPSA for the equilibrium equation [Eq. (21)]. Top: The dashed green line (marked RPSA) indicates the values of $\left\langle\phi^{\mathrm{RPSA}}\right\rangle_{0}$ in Eq. (23). The red solid line (marked num) shows the full numerical result $\left\langle\phi^{\text {num }}\right\rangle_{0}$ from the time evolutions. Bottom: Moments obtained from the numerical time evolutions. The simulation size is $L=128$, and the duration of the time evolution is $t_{\max }=300$. Other parameters are $\alpha=3.5, \beta=2.0$, and $\gamma=2 / \pi$. The external field parameters are $B_{0}=1.5$ and $v_{\mathrm{B}}=0.01$.

\section{PHASE-PREDICTION METHOD BY THE RPSA EQUILIBRIUM EQUATION}

The categorization of the RPSA equilibrium equations described in Sec. VII is applicable in phase predictions of the TDGL dynamics. We utilize Table I to predict the corresponding phase for each point $(\alpha, \beta, \gamma, A)$ in the parameter space. The continuum equation of motion [Eq. (11)] is employed, enabling us to use the analytic form of Green's function shown in Eq. (10). Note that this choice causes a modification to the definition of $Q_{k}$, as follows:

$$
Q_{k}=\frac{\alpha-\beta|\boldsymbol{k}|^{2}-\gamma(2 \pi)^{2} G_{k}(A)}{\alpha},
$$

where $G_{k}(A)$ represents the continuum limit of Green's function shown in Eq. (10). The method "PhasePrediction" is schematically shown below. This is a procedure that maps the system parameters $(\alpha, \beta, \gamma, A)$ to the output Phase $\in\{$ Symmetric, T-breaking, $*$ Z-breaking $\}$, with $*$ Z-breaking meaning either TZ-breaking or Z-breaking.

1: procedure PHASEPREDICTION $(\alpha, \beta, \gamma, A)$

2: $g(k)=\left(1-\mathrm{e}^{-A k}\right) /\left(\pi A^{2} k\right)$

3: $Q(k)=1-\alpha^{-1} \beta k^{2}-\alpha^{-1} \gamma(2 \pi)^{2} g(k)$

4: if $Q(0)<0$ then

5: $\quad$ if $\max (Q)<0$ then

6: $\quad$ Phase $\Leftarrow$ Symmetric

7: $\quad$ else

8: $\quad$ Phase $\Leftarrow$ T-breaking

9: $\quad$ end if

10: else
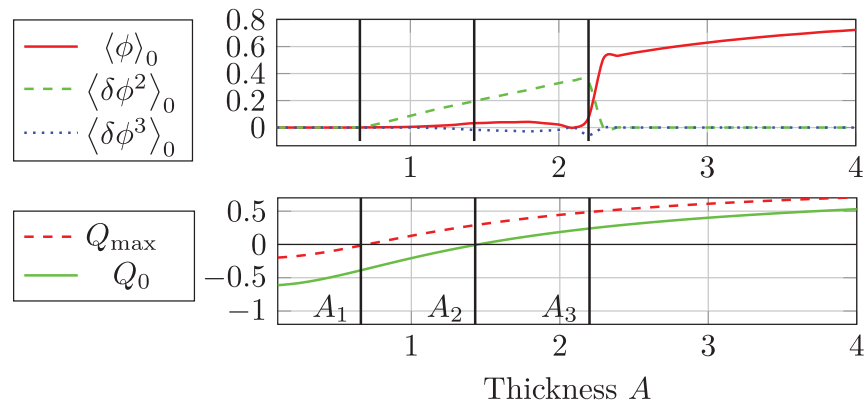

FIG. 3. Top: Thickness dependences of the moments in the final spin configuration (same as Fig. 2). Bottom: $Q_{\max }\left[=\max \left(Q_{k}\right)\right]$ and $Q_{0}$, directly obtained from the Green's function without the time evolutions. The numerical setup is similar to that shown in Fig. 2.

\section{1: $\quad$ Phase $\Leftarrow *$ Z-breaking \\ 12: end if \\ 13: end procedure}

Using this method, we can predict the phases for each point $(\alpha, \beta, \gamma, A)$. As an example, let us investigate the series of numerical simulations shown in Fig. 3. In this example, we increase the value of $A$ from 0.1 to 4 , while the other parameters remain fixed to the original values. In the first region $0<A<A_{1}(\sim 0.7)$, we have negative values for $Q_{0}$ and $Q_{k}$ for any $\boldsymbol{k}$. In this region, $\langle\phi\rangle_{0}$ is always zero. Owing to the equilibrium equation, Eq. (22), we also conclude that $\phi_{k}=0$ for any $\boldsymbol{k}$. This is the symmetric phase. Once the thickness exceeds the value $A=A_{1}$, the coefficient $Q_{k}$ becomes nonnegative for some $\boldsymbol{k}$ points because $Q_{\max }[=\max (Q)]$ exceeds zero, whereas $Q_{0}$ remains negative (Fig. 3). This results in nonzero values for a limited number of modes, as Eq. (22) is now satisfied for modes $\langle\phi\rangle_{k}$, so that $Q_{k}-3\left\langle\delta \phi^{2}\right\rangle_{0}=0$ (note that $\left.\left\langle\delta \phi^{2}\right\rangle_{0} \geqslant 0\right)$. The zero wave number mode $\langle\phi\rangle_{0}$ is still zero (T-breaking phase). If the value of $A$ is further increased, $Q_{0}>0$ is obtained for $A>A_{2}(\sim 1.4)$. This causes a nonzero value of $\langle\phi\rangle_{0}$, which spreads into several modes via Eq. (22). This is the TZ-breaking phase. After a certain value, $A=A_{3}$ $(\sim 2.2)$, the domain structure vanishes because the Z-breaking phase becomes stable. Note that the large aspect ratio in the T-breaking phase is partly explained by the small number of nonzero modes.

\section{DISCUSSION}

Figure 4 shows the phase diagram for the normalized TDGL dynamics [Eq. (12)] predicted by phase prediction. The overall tendency matches our physical instincts well. As $p_{1}$ becomes large, the demagnetization effect from the dipole-dipole interactions supersedes the anisotropy to yield the symmetric phase, while for large values of $p_{4}$, it is partly relaxed by the thickness to have more complex structures.

We also compared the numerical results of the time evolution with those of the phase prediction (see Table II for a comparison). The computational cost for phase prediction is negligible compared to that for the corresponding numerical time evolutions. The agreement between the time evolution and phase prediction is good, except for the cases $\left(p_{1}, p_{4}\right)=$ 

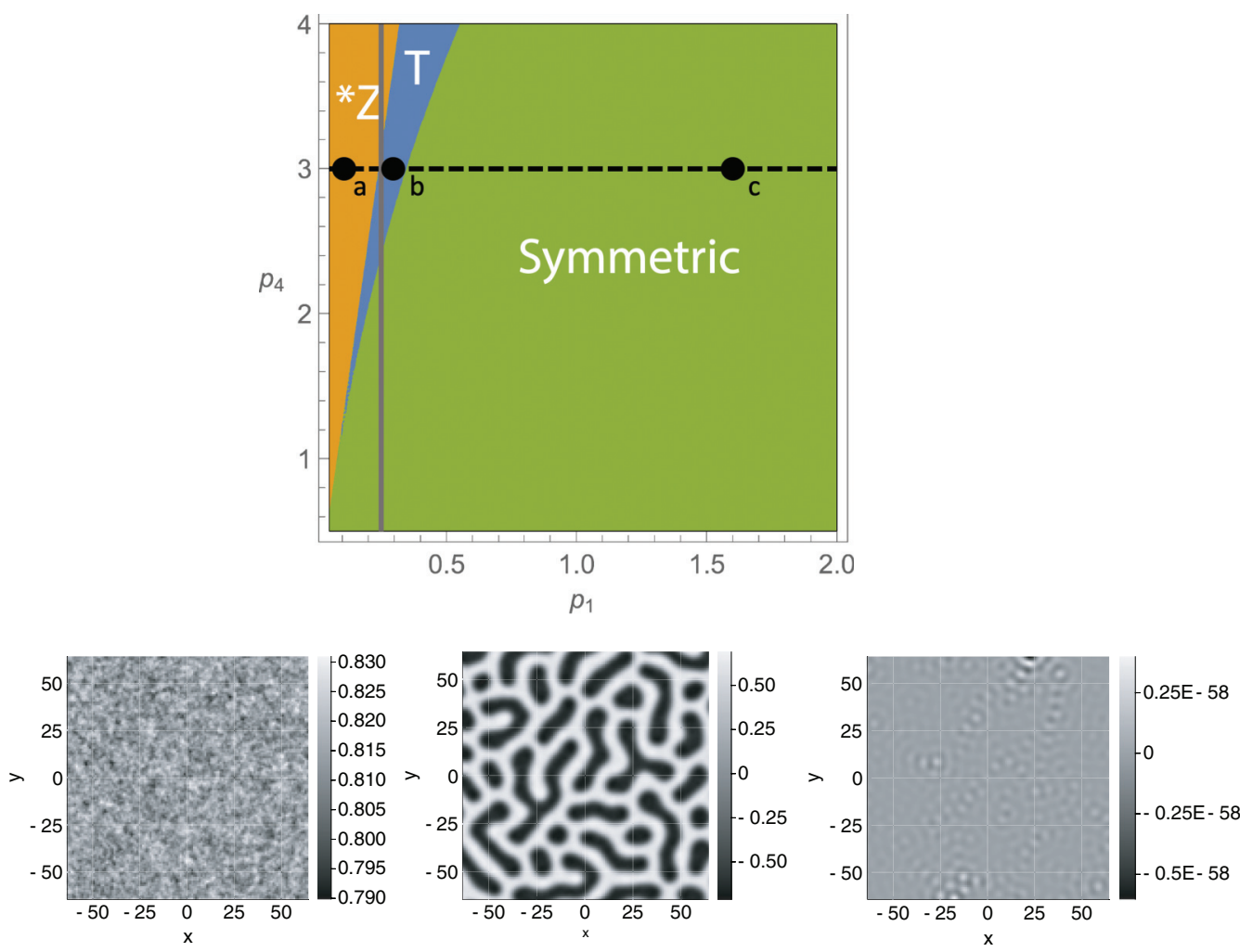

FIG. 4. Top: The phase diagram of the TDGL dynamics corresponding to Eq. (12) estimated using the phase prediction method proposed in Sec. VIII. Orange area (marked *Z), TZ- or Z-breaking phase; blue area (T), T-breaking phase; green area (Symmetric), symmetric phase. The gray solid line shows the contour of the normalized parameters corresponding to the system parameters used in Figs. 2 and 3 . Bottom: Patterns corresponding to points $\mathrm{a}, \mathrm{b}$, and $\mathrm{c}$ on the phase diagram above. The expected patterns corresponding to the phases were obtained. Note that point $\mathrm{c}$ shows a pattern with $\phi(\boldsymbol{r})=0$ for all $\boldsymbol{r}$, which is the definition of the symmetric phase.

$(0.1,1.0),(0.4,2.5),(0.4,3.0)$. This is due to the relatively small absolute values of $\max (Q)$ and $Q(0)$ at these sample points. Because the RPSA neglects the third-order moments in the equilibrium equations, the results obtained using the RPSA equilibrium equations may differ from the time evolution for small $|Q(0)|$ and $|\max (Q)|$.

Note that the external magnetic sweep rate $v_{\mathrm{B}}$ is a crucial parameter in pattern formation. It has been reported [7] that

TABLE II. Comparison of numerical time evolutions with the phase prediction method. T, Z, TZ, and S denote the phases observed from the time evolution (T-, Z-, and TZ-breaking and symmetric phases, respectively), while ( $s, t)$ with $s, t \in\{+,-\}$ denote the signs of $Q_{0}$ and $\max (Q)$, respectively. Note that phase prediction translates $(++)$ to $* \mathrm{Z}(=\mathrm{Z}, \mathrm{TZ}),(-+)$ to $\mathrm{T}$, and $(--)$ to $\mathrm{S}$. The numerical time evolutions are performed for the system with $512^{2}$ grid points using the normalized TDGL equation, Eq. (12). Points marked with a star $(\star)$ indicate mismatches with the numerical results.

\begin{tabular}{lccccc}
\hline \hline & \multicolumn{5}{c}{$p_{1}$} \\
\cline { 2 - 6 }$p_{4}$ & 0.1 & 0.2 & 0.4 & 0.8 & 1.6 \\
\hline 1.0 & $\mathrm{Z}^{\star}(--)$ & $\mathrm{S}(--)$ & $\mathrm{S}(--)$ & $\mathrm{S}(--)$ & $\mathrm{S}(--)$ \\
1.5 & $\mathrm{Z}(++)$ & $\mathrm{T}(--)$ & $\mathrm{S}(--)$ & $\mathrm{S}(--)$ & $\mathrm{S}(--)$ \\
2.0 & $\mathrm{Z}(++)$ & $\mathrm{TZ}(-+)$ & $\mathrm{S}(--)$ & $\mathrm{S}(--)$ & $\mathrm{S}(--)$ \\
2.5 & $\mathrm{Z}(++)$ & $\mathrm{Z}(++)$ & $\mathrm{T}^{\star}(--)$ & $\mathrm{S}(--)$ & $\mathrm{S}(--)$ \\
3.0 & $\mathrm{Z}(++)$ & $\mathrm{Z}(++)$ & $\mathrm{T}^{\star}(--)$ & $\mathrm{S}(--)$ & $\mathrm{S}(--)$ \\
\hline \hline
\end{tabular}

domain formation is largely affected by $v_{\mathrm{B}}$. Our results here must be understood as an approximated result, not only in the RPSA but also in eliminating the effects of the magnetic sweep rate. For large values of $v_{\mathrm{B}}$, the time evolution has similar results, whereas for small values of $v_{\mathrm{B}}$, it can be quite different. For details, see Appendix A.

As shown in this section, for $0.1 \leqslant p_{1} \leqslant 1.6$ and $1.0 \leqslant$ $p_{4} \leqslant 3.0$, phase prediction shows good concordance with the numerical time evolutions without any fitting parameters or assumptions of domain patterns.

\section{CONCLUSIONS}

The long history of research on magnetism and the mathematical structure of the TDGL dynamics shows a wide variety of approaches to pattern formation in magnetic materials [18-22].

Although most existing methods use artificial functions that specify the magnetic domain patterns, we focus on the equilibrium equation that a magnetic material must satisfy in its equilibrium state. The application of the RPSA [29] to the equilibrium equations enables us to predict the phase in the equilibrium state. The prediction matches the actual numerical time evolution results qualitatively without any fitting parameters. Although the prediction is not perfect, our method involves no a priori assumptions of the domain patterns, e.g., stripe [21] or stripe and check [19]. Our method is based on the equilibrium equation approximated via the RPSA. RPSA 

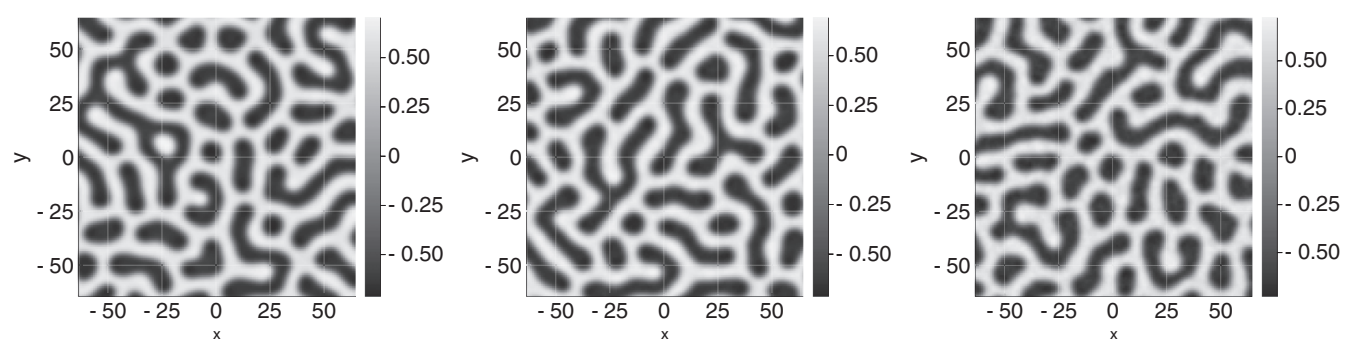

FIG. 5. The numerical simulations for different values of the standard deviation $\sigma$. Left: $\sigma=0.15$. Middle: $\sigma=0.3$ (original). Right: $\sigma=0.6$. System parameters: $\left(\alpha, \beta, \gamma, B_{0}, v_{\mathrm{B}}\right)=(1,1,0.3,1.5,0.01)$ with $L=512$.

factorizes the higher moments to compress the numerically heavy terms, which are also difficult to treat analytically. Because it simply factorizes the higher moments of the field $\delta \phi$, it implements no fitting parameters. Our method also has no dependences on the initial domain patterns.

As a future work, we can extend our method by using the Fourier mode decomposition in the time region of the spin variables to deal with the spin system under periodic external fields. Such an extension of this method enables us to predict a wider variety of physical characteristics, e.g., dynamic susceptibility and the memory effect [6] in magnetic materials.

Moreover, the object of this method is not limited to magnetic systems. It is applicable in a vast class of natural/social phenomena that seemingly have nothing in common but can be described by equations of motion, such as Eqs. (1) and (2).

One such application is parameter estimation in material and statistical physics. Using Bayesian inference methods, we can estimate the parameters of a system with large degrees of freedom using relatively little observation/numerical data (e.g., [30]). Our method is expected to work for such parameter estimations in various systems on a theoretical and numerical basis by providing information on the phase for each parameter using negligible computational costs, with physically justifiable reasons.

\section{ACKNOWLEDGMENTS}

This study was mainly supported by JST CREST Grants No. JPMJCR1761 and No. JPMJCR1861 and partially supported by Grant No. JPMJCR1763 of the Japan Science and Technology Agency. The key ideas in this study emerged through the activities of JSPS KAKENHI Grants No. JP19K14671, No. JP17H01703, No. JP17H01704, No. JP18H03210, No. JP19H05662, and No. JP20K21785. The travel expenses needed to facilitate discussion among coauthors were partially supported by ERI JURP Grants No. ERI JURP 2020-A-05, No. ERI JURP 2018-B-01, and No. ERI JURP 2019-B-04. This study was also partially supported by the Council for Science, Technology and Innovation, Crossministerial Strategic Innovation Promotion Program (SIP), "Structural Materials for Innovation" (funding agency: JST).

\section{APPENDIX A: EXTERNAL FIELD SWEEP RATE}

The effects of the external field sweep rate on the TDGL dynamics of the kinetic Ising model were extensively studied by Kudo and Nakamura [7]. Specifically, the average domain size of the final pattern and other important features were found to be affected by the sweep rate. In this study, we focus on the fast quench regime with $p_{3}>8 \times 10^{-4}$. Faster sweep rates are confirmed to have no effects on the final results, although slower sweep rates may result in qualitatively different final (equilibrium) domain patterns. We use the ReLU function $R(x)=\max (x, 0)$ to realize a constant sweep rate.
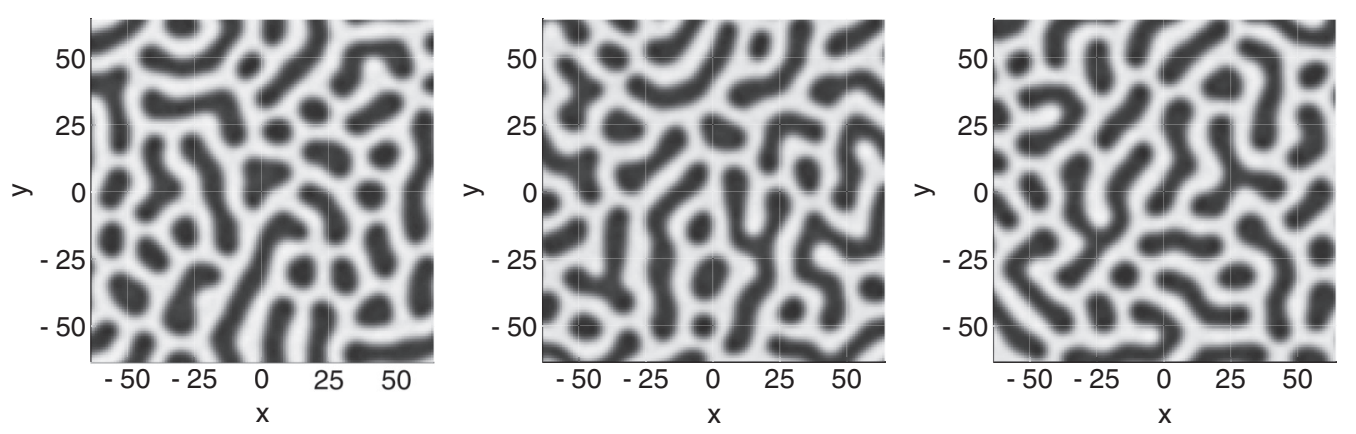

FIG. 6. Size dependences of the numerical simulations. The two-dimensional magnetization pattern in square regions with a size of $128 \times$ 128 [for $L=256$ and 512, the depicted areas are cropped around the center $(x, y)=(0,0)$ of the entire simulation]. System parameters: $\left(\alpha, \beta, \gamma, B_{0}, v_{\mathrm{B}}\right)=(1,1,0.3,1.5,0.01)$ with $L=128$ (left), 256 (middle), and 512 (right). 
Fig. 4. The line crosses the most complicated area in the phase diagram and has approximately 30 points in the depicted area. Thus, the relevance of RPSA is reasonably checked by the system parameters used in Sec. VII.

The roughness of the material is represented by the random variable $\Lambda(\boldsymbol{r})=1+\lambda(\boldsymbol{r}) / 4$ with the Gaussian noise $\lambda(\boldsymbol{r}) \sim$ $\mathcal{N}\left(0, \sigma^{2}\right)$ independently and identically for all $\boldsymbol{r}$ in Sec. III. The standard deviation of the Gaussian noise $\sigma$ is set to 0.3 in the main text. Here, we also show the results for $\sigma=$ 0.15 and $\sigma=0.6$ in Fig. 5. No qualitative difference was observed.

\section{APPENDIX C: SYSTEM SIZE}

The system size $L$ is also a vital parameter, which may affect the numerical results. We mostly used a system with $L=$ 512 (i.e., a square system where the number of grid points is $\left.512^{2}=262,144\right)$. A smaller system, with $L=128$, was used only for the numerical tests concerning the non-RPSA effects in Sec. VII. The numerical results for the system parameters $\left(\alpha, \beta, \gamma, B_{0}, v_{\mathrm{B}}\right)=(1,1,0.3,1.5,0.01)$ with $L=128,256$, 512 are shown in Fig. 6. There are no size dependences observed in this simulation. The temporal profiles of the average magnetization also have no size dependences for $L \geqslant 128$.
[1] J. H. Van Vleck, Rev. Mod. Phys. 17, 27 (1945).

[2] C. Kittel, Rev. Mod. Phys. 21, 541 (1949).

[3] M. Suzuki et al., J. Phys.: Conf. Ser. 430, 012017 (2013).

[4] P. N. Argyres, Phys. Rev. 97, 334 (1955).

[5] J. McCord, J. Phys. D 48, 333001 (2015).

[6] E. A. Jagla, Phys. Rev. E 70, 046204 (2004).

[7] K. Kudo and K. Nakamura, Phys. Rev. B 76, 054111 (2007).

[8] K. Iwano, C. Mitsumata, and K. Ono, J. Appl. Phys. 115, 17D134 (2014).

[9] K. Kawasaki, Prog. Theor. Phys. 52, 359 (1974).

[10] K. Kawasaki, Prog. Theor. Phys. 51, 1064 (1974).

[11] K. Kawasaki, Prog. Theor. Phys. 52, 84 (1974).

[12] M. Suzuki and G. Igarashi, Prog. Theor. Phys. 49, 1070 (1973).

[13] M. Grant, M. San Miguel, J. Vials, and J. D. Gunton, Phys. Rev. B 31, 3027 (1985).

[14] K. Kawasaki and T. Ohta, Phys. A (Amsterdam, Neth.) 116, 573 (1982).

[15] T. Yasui, H. Tutu, M. Yamamoto, and H. Fujisaka, Phys. Rev. E 66, 036123 (2002).

[16] N. Fujiwara, H. Tutu, and H. Fujisaka, Phys. Rev. E 70, 066132 (2004).
[17] T. M. Rogers, K. R. Elder, and R. C. Desai, Phys. Rev. B 37, 9638 (1988).

[18] C. Kittel, Phys. Rev. 70, 965 (1946).

[19] B. Kaplan and G. Gehring, J. Magn. Magn. Mater. 128, 111 (1993).

[20] G. Bochi, H. J. Hug, D. I. Paul, B. Stiefel, A. Moser, I. Parashikov, H.-J. Güntherodt, and R. C. O'Handley, Phys. Rev. Lett. 75, 1839 (1995).

[21] C. Kooy and U. Enz, Philips Res. Rep. 15 (1960).

[22] A. Lisfi and J. C. Lodder, J. Phys.: Condens. Matter 14, 12339 (2002).

[23] T. Garel and S. Doniach, Phys. Rev. B 26, 325 (1982).

[24] K. Kudo, M. Mino, and K. Nakamura, J. Phys. Soc. Jpn. 76, 013002 (2007).

[25] T. Yokota, J. Magn. Magn. Mater. 432, 532 (2017).

[26] T. Tomé and M. J. de Oliveira, Phys. Rev. A 41, 4251 (1990).

[27] S. Krogstad, J. Comput. Phys. 203, 72 (2005).

[28] C. B. Muratov, Phys. Rev. E 66, 066108 (2002).

[29] R. Anzaki, K. Fukushima, Y. Hidaka, and T. Oka, Ann. Phys. (NY) 353, 107 (2015).

[30] S. Ito, H. Nagao, T. Kurokawa, T. Kasuya, and J. Inoue, Phys. Rev. Mater. 3, 053404 (2019). 